\title{
A Field Study on the Barriers in the Assimilation of Open Source Server Software
}

\author{
Kris Ven and Jan Verelst \\ Department of Management Information Systems, \\ University of Antwerp, Antwerp, Belgium \\ $\{$ kris.ven, jan.verelst\}@ua.ac.be
}

\begin{abstract}
An increasing number of academic studies have been devoted to the organizational adoption of open source software (OSS). Most studies have either focused on determining which reasons influence the adoption of OSS, or which barriers prevent the adoption of OSS. To our knowledge, no prior study has been conducted to determine which barriers exist to the further adoption of OSS in organizations. Studies addressing this issue could provide more insight into whether organizations are effectively able to overcome the initial barriers to adoption, or whether new barriers arise when the organization expands its use of OSS. To this end, we conducted a qualitative field study involving 56 organizations that were asked to report on which barriers existed to their further adoption of OSS. The data was analyzed using a mixed methods approach by combining both qualitative and quantitative techniques. Results showed that the main barrier reported by organizations was a lack of internal and external knowledge. Furthermore, our results indicate that there was no relationship between the barriers reported by organizations and their extent of OSS usage. This indicates that these barriers remain important as organizations increase their assimilation of OSS.
\end{abstract}

Keywords: open source software, adoption, assimilation, knowledge, barriers.

\section{Introduction}

In the past few years, an increasing number of academic studies have been devoted to the organizational adoption of open source software (OSS). One line of research has investigated which reasons influence the adoption of OSS (see e.g., 3281418161531 ). These studies have provided more insight into which factors have a positive impact on the adoption of OSS. Typically, such studies hypothesize that organizations that exhibit a number of favorable characteristics are more likely to exhibit a greater extent of adoption [6]. Hence, these studies consider both organizations with a low extent of adoption and organizations with a high extent of adoption. A second line of studies has investigated the non-adoption of OSS (see e.g., 2012513191011]). These studies are concerned with determining which barriers prevent organizations from adopting 
OSS. These barriers can have an important negative influence on the organizational adoption of OSS. It is important to realize that adoption and non-adoption are indeed two distinct phenomena 219. Gatignon and Robertson have noted that "the variables accounting for rejection are somewhat different from those accounting for adoption; rejection is not the mirror image of adoption, but a different form of behavior." [9, p. 42]. For example, it has been shown that even in the presence of several strong arguments in favor of the adoption of OSS, the existence of additional factors with a negative impact on adoption may result in non-adoption [13. Previous studies that investigate the barriers to the adoption of OSS generally use a sample of organizations that have not adopted OSS (e.g., 101113 ).

To our knowledge, no prior study has been conducted to determine which barriers exist to the further adoption of OSS in organizations. However, we argue that it is interesting to consider which barriers exist for organizations that have already adopted OSS to some extent. These barriers may limit the ability of organizations to further increase their adoption of OSS. It seems unlikely that an organization that has adopted OSS to a limited extent will no longer experience any barriers when expanding its use of OSS. Instead, a more gradual process is likely to take place in which organizations slowly overcome the barriers to adoption over time. Hence, there is currently a lack of studies that consider the impact of barriers to the further adoption of OSS. Studies addressing this issue could provide more insight into whether organizations are effectively able to overcome the initial barriers to adoption, or whether new barriers arise when the organization expands its use of OSS. The results from such studies could allow to devise interventions that facilitate the further adoption of OSS in organizations.

In this study, we will address this gap in literature by investigating how the barriers reported by organizations evolve as organizations increase their use of OSS. To this end, a qualitative field study involving 56 organizations was conducted to determine which barriers existed to the further adoption of OSS. Our sample included organizations that did not adopt OSS, as well as organizations that have adopted OSS to at least some extent. Each respondent was asked to report which barriers they perceived to be present to the further adoption of OSS. We investigated whether the existence of these barriers was related to the extent to which the organization has adopted OSS. This will provide insight into whether these barriers are overcome by organizations as they increase their assimilation of OSS, and which additional barriers may arise later in the adoption process.

\section{Methodology}

Our study was conducted as a qualitative field study. A self-administered web survey was used to collect the data for our study. The scope of our survey was restricted to the use of open source server software (OSSS). For the purpose of this survey, the term OSSS referred to a limited list of 7 OSS products consisting of Linux, BSD, Apache, Bind, Sendmail, Postfix and Samba. Respondents were 
instructed on each page of the survey that the term OSSS referred to this specific list of OSS products. Our sample consisted of Belgian organizations from different sectors and sizes. The target person in each organization was the IT decision maker, commonly the CIO or IT manager. We received a reply from 111 out of 153 organizations that were sent an invitation to participate, which corresponds to a response rate of $72.5 \%$.

The main question in the survey that respondents needed to answer was the following: "Which reasons prevent the (further) adoption of OSSS in your organization?". The question was open-ended, allowing organizations to provide a free format reply. This approach allowed us to obtain more in-depth information than by using a closed-ended question. In addition, organizations were also asked to report on their extent of Linux adoption and their degree of OSSS assimilation. The extent of Linux adoption was measured on a 7-point point Likert scale ranging from "no usage" to "to a very large extent". The assimilation of OSSS was measured by using the Guttman scale developed by Fichman and Kemerer [7. which was slightly reworded to fit the context of the adoption of OSSS (see also 32 ).

A mixed methods approach was used to analyze our data, by using both qualitative and quantitative techniques 2422. In a first step, our data was analyzed by using qualitative techniques 17227 . This analysis was performed by using NVivo 8. After importing the data in NVivo, all replies were coded to identify any barriers reported by respondents. In a first cycle, coding took an inductive approach by creating new codes as new reasons were identified in the responses [2 27]. After this first cycle, a list of 21 codes was obtained. In a second cycle, the codes were further aggregated into a hierarchy consisting of 8 main categories representing the barriers to the further adoption of OSSS [2 27].

In a second step, the qualitative data was converted into quantitative data. This process is commonly referred to in mixed methods research as quantitizing 2423328 . After quantitizing, statistical methods can be used to test hypotheses regarding the relationships between independent and dependent variables derived from both qualitative and quantitative data 28 3. Combining qualitative and quantitative analysis techniques is an accepted approach in mixed methods research and can assist the researcher to more easily distinguish and communicate patterns within the qualitative data [242332817]. Each barrier that was coded in the qualitative analysis was converted into a dichotomous variable by using NVivo. Each case was assigned a value of one if the barrier was present, and zero otherwise. This resulted in a matrix in which the presence or absence of each code derived from the qualitative data was recorded for each case. This matrix is referred to as an inter-respondent matrix or a case-by-variable matrix 243 . This matrix was further extended with quantitative data concerning the degree of OSSS assimilation and the extent of Linux adoption. This matrix was then used to perform statistical analyses to further analyze the data [24/233 28]. These analyses were performed in SPSS 15.0. 


\section{Findings}

A quick inspection of our data showed that 55 out of 111 respondents did not provide an answer to the question of which barriers (further) inhibit the use of OSSS. This was indicated by an empty string for the reply field. A missing reply could have two causes: (1) the respondent did not reply to the question, or (2) the respondent could not identify any barriers to the further adoption of OSSS. Unfortunately, we were not able to make a distinction between these two scenarios. All cases with empty responses were therefore coded as unanswered. These cases were discarded in the further analysis of our data. As a result, we obtained usable replies from 56 organizations in our sample. We will first present an overview of which barriers were reported by our respondents. Next, we will investigate whether these barriers are related to the assimilation of OSSS or the extent of Linux adoption.

\subsection{Barriers to the Further Adoption of OSSS}

A general overview of which barriers were mentioned by the organizations in our sample is presented in Table 1] This table contains the 8 barriers that were identified during the coding process. One barrier ("insufficient knowledge") is further divided into two subcategories ("internal" and "external" knowledge). The second column indicates how many organizations have reported each barrier 11 The final column indicates the percentage of organizations that has reported each barrier. Since organizations could mention more than one barrier, the total of this column does not add up to $100 \%$. This column represents the frequency effect size of each barrier [22]. We will now discuss each of these barriers in more detail.

Insufficient Knowledge. The most important barrier that was mentioned by almost a third of the organizations is a lack of knowledge about OSSS. We further distinguished between insufficient internal knowledge (i.e., knowledge that is held by employees within the organization) and external knowledge (i.e., knowledge that is offered by service providers). Our data shows that internal knowledge is the most important component. Many organizations reported that they lacked employees that are familiar with OSSS. One respondent mentioned that it was "difficult to find in-house the right technical staff to maintain [OSSS]". In addition, the knowledge base within the organization may also restrict the opportunities to explore the use of OSSS. One respondent mentioned that the increased use of OSSS was difficult due to "colleagues who are more proprietary-oriented". With respect to the availability of external knowledge, organizations were concerned that no commercial support was available for OSSS and that the internal IT staff would be responsible for supporting the software. One respondent noted

\footnotetext{
${ }^{1}$ Since one organization reported a lack of both internal and external knowledge, the number of organizations that reported insufficient knowledge is not the sum of the number of organizations that reported insufficient internal and external knowledge.
} 
Table 1. Overview of Barriers Mentioned by Respondents

\begin{tabular}{lrr}
\hline Rank Barrier & $\begin{array}{c}\text { Number of } \\
\text { Organizations }\end{array}$ & $\begin{array}{c}\text { Percentage of } \\
\text { Organizations }\end{array}$ \\
\hline 1. Insufficient Knowledge & 18 & $32.1 \%$ \\
Insufficient internal knowledge & 13 & $23.2 \%$ \\
Insufficient external knowledge & 6 & $10.7 \%$ \\
2. No further barriers & 15 & $26.8 \%$ \\
3. Limited functionality & 9 & $16.1 \%$ \\
4. Management guidelines & 8 & $14.3 \%$ \\
5. Insufficient resources & 7 & $12.5 \%$ \\
6. Dependency on vendor & 3 & $5.4 \%$ \\
7. Not a goal & 2 & $3.6 \%$ \\
8. Satisfaction with proprietary software & 1 & $1.8 \%$ \\
Total organizations responding: & 56 & \\
\hline
\end{tabular}

Note: percentages do not add up to $100 \%$ since organizations could mention more than one barrier.

that "currently no suppliers offer/support OSSS". It was verified that this specific respondent was not interested in using OSSS in the organization, and can therefore be expected to have accumulated little knowledge about OSSS.

No Further Barriers. Some organizations explicitly mentioned that they could not identify any barriers to the further adoption of OSSS2 Although this is not a real barrier that inhibits the use of OSS, it is interesting to consider this factor as it identifies those organizations that have overcome all barriers to adoption. For one organization, the absence of any remaining barriers appeared to have paved the way to make OSSS the preferred technology for the organization, as the respondent wrote: "We don't have any reason to prevent future use of OSSS! We have the intention to use OSSS as much as possible!".

Limited Functionality. Several organizations mentioned the limited functionality of OSSS as a major barrier to adoption. The most important remark in this regard was the poor interoperability with proprietary applications. For example, one respondent mentioned "compatibility issues on file-level and application-level with Microsoft software". One organization from the financial sector also raised concerns with respect to the "necessary security requirements in a financial environment".

Management Guidelines. The attitude of managers may also impede the use of OSSS. Several respondents expressed that managers in the organization were either "unease about the use of OSS" or simply "not in favor of OSS". This

${ }^{2}$ Please note that we were not able to determine whether a blank reply meant that the respondent did not reply to the question, or that the respondent could not identify any barriers. It is therefore likely that our data slightly underestimates the number of respondents that could not identify any barriers. 
appeared to be mainly due to a lack of trust in OSS. As one respondent mentioned: "we work for a large organization who values the trusted vendors more than the cost savings of working with $O S S^{\prime \prime}$. As a result, some organizations had a clear policy that did not allow the use of OSSS. The respondent in the financial organization mentioned above, for example, indicated that "an in-house policy forbids the use of all OSSS". In organizations in which IT plays an important role, managers may also prefer to posit strict guidelines with respect to the use of IT. This was illustrated by one respondent as: "the decision of the board to go with 'standardization', which seems to be Microsoft nowadays".

Insufficient Resources. Some organizations reported that a lack of resources (i.e., time, money, and human resources) inhibited the use of OSSS. For most organizations, the main issue in this respect seemed to be a lack of internal resources that are available to gain experience with emerging technologies. This was expressed by one respondent as: "not enough manpower to try out new things". One respondent considered OSSS unsuitable for organizational use by saying that: "we have no time for such hobbies". Another organization seemed to think that using OSSS still required much customization efforts by explaining that the use of OSSS would require "too much investment to make tailor-made".

Dependency on Vendor. Some organizations mentioned that they could not adopt OSSS because they fully depended on a vendor for their software. Interestingly, this factor was mentioned by three public organizations, all so-called Public Center for Social Welfare (PCSW). As expressed by one respondent, "given the specific PCSW software, we are dependent on our software vendor". These organizations need to use specific software for their services, and only a limited number of vendors currently offer this software on the market.

Not a Goal. Two organizations mentioned that the further use of OSSS was not a goal in itself. One respondent indicated that the further use of OSSS was "not a necessity". This seems to suggest that these organizations do not want to increase the use of OSSS per se, but rather decide on a project-per-project basis which solution (proprietary or OSS) is best suited.

Satisfaction with Proprietary Software. Finally, one organization mentioned that "we are satisfied with our current proprietary software [operating system] and don't see the ROI or the major change in TCO to move to OSSS". If organizations are satifisfied with their existing systems, there may indeed be no compelling reason to consider the adoption of OSS.

\subsection{Relationship with Assimilation of OSSS}

Next, we determined whether the existence of certain barriers was related to the assimilation stage reached by the organization. The Guttman scale developed by Fichman and Kemerer [7] classifies organizations into 7 different assimilation stages. Organizations that are situated in stages 0 to 3 have not yet made a formal decision to adopt OSSS. They have progressed at most to the trial or 
Table 2. Relationship between Assimilation and Barriers Reported

\begin{tabular}{|c|c|c|c|c|c|}
\hline \multirow[b]{2}{*}{ Barrier } & \multicolumn{2}{|c|}{$\begin{array}{c}\text { Number of } \\
\text { organizations }\end{array}$} & \multicolumn{2}{|c|}{$\begin{array}{l}\text { Percentage of } \\
\text { organizations }\end{array}$} & \multirow{2}{*}{$\begin{array}{c}\text { Fisher } \\
\text { Exact } \\
\text { Test }\end{array}$} \\
\hline & NAD & $\overline{A D}$ & NAD & AD & \\
\hline 1. Insufficient knowledge & 10 & & $37.0 \%$ & $27.6 \%$ & .319 \\
\hline Internal knowledge & 7 & & $25.9 \%$ & $20.7 \%$ & .441 \\
\hline External knowledge & 4 & & $14.8 \%$ & $6.9 \%$ & .301 \\
\hline 2. No further barriers & 5 & 10 & $18.5 \%$ & $34.5 \%$ & .148 \\
\hline 3. Limited functionality & 4 & & $14.8 \%$ & $17.2 \%$ & .547 \\
\hline 4. Management guidelines & 3 & & $11.1 \%$ & $17.2 \%$ & .395 \\
\hline 5. Insufficient resources & 5 & & $18.5 \%$ & $6.9 \%$ & .182 \\
\hline 6. Dependency on vendor & 1 & 2 & $3.7 \%$ & $6.9 \%$ & .527 \\
\hline 7. Not a goal & 2 & 0 & $7.4 \%$ & $0.0 \%$ & .228 \\
\hline 8. Satisfaction with proprietary software & 1 & 0 & $3.7 \%$ & $0.0 \%$ & .482 \\
\hline Total number of organizations: & 27 & 29 & & & \\
\hline
\end{tabular}

Note: percentages do not add up to $100 \%$ since organizations could mention more than one barrier.

Legend: NAD: non-adopter, AD: adopter.

evaluation stage, where the use of OSSS is still being considered. Organizations in the latter stages (4 to 6) have made a formal decision to adopt, and are using OSSS in a production environment. It therefore appears that stage 4 is a logical boundary between assimilation stages. For the purpose of this analysis, organizations that have not progressed beyond stage 3 (evaluation/trial) will be called "non-adopters", while organizations that have reached at least stage 4 will be called "adopters".

Table 2 shows how many adopters and non-adopters have mentioned each barrier. Similar to Table 1, we also calculated the frequency effect size for each barrier [22]. This effect size was calculated by dividing the number of non-adopters (or adopters) that mentioned a specific barrier by the total number of nonadopters (or adopters). These effect sizes are displayed in column 4 and 5 and indicate the relative importance of each barrier for each group.

In order to test whether there are statistically significant differences between the barriers reported by adopters and non-adopters, we constructed a $2 \times 2$ contingency table for each of the barriers by using the data from Table 2. The rows classified organizations according to whether they mentioned a specific barrier, while the columns distinguished between adopters and non-adopters. The Fisher Exact Test was used to test for significant relationships. The Fisher Exact Test can be used when the assumptions of the $\chi^{2}$-test are violated [26]. The use of this test was appropriate in this case given the use of a $2 \times 2$ table, the relatively small sample for each barrier, and an expected frequency below five for some cells [26]. The Fisher Exact Test returns the propability that exactly the same observed distribution or an even more disproportional distribution would be obtained by taking into account the row and column totals [26]. A one-tailed, directional test was performed since it is expected that adopters would report 
Table 3. Relationship between Linux Adoption and Barriers Reported

\begin{tabular}{lrrrrr}
\hline & \multicolumn{3}{c}{$\begin{array}{c}\text { Number of } \\
\text { organizations }\end{array}$} & \multicolumn{3}{c}{$\begin{array}{c}\text { Percentage of Fisher } \\
\text { organizations }\end{array}$} & $\begin{array}{c}\text { Exact } \\
\text { Barrier }\end{array}$ & NLU & LU & NLU & LU & Test \\
\hline 1. Insufficient knowledge $\quad 13$ & 5 & $39.4 \%$ & $21.7 \%$ & .499 \\
$\quad$ Internal knowledge & 9 & 4 & $27.3 \%$ & $17.4 \%$ & .220 \\
$\quad$ External knowledge & 5 & 1 & $15.2 \%$ & $4.3 \%$ & .665 \\
2. No further barriers & 6 & 9 & $18.2 \%$ & $39.1 \%$ & .381 \\
3. Limited functionality & 5 & 4 & $15.2 \%$ & $17.4 \%$ & .063 \\
4. Management guidelines & 5 & $315.2 \%$ & $13.0 \%$ & .503 \\
5. Insufficient resources & 6 & 1 & $18.2 \%$ & $4.3 \%$ & .418 \\
6. Dependency on vendor & 0 & 3 & $0.0 \%$ & $13.0 \%$ & .512 \\
7. Not a goal & 1 & 1 & $3.0 \%$ & $4.3 \%$ & .643 \\
8. Satisfaction with proprietary software & 1 & 0 & $3.0 \%$ & $0.0 \%$ & .804 \\
\multicolumn{1}{c}{ Total number of organizations: } & 33 & 23 & & & \\
\hline
\end{tabular}

Note: percentages do not add up to $100 \%$ since organizations could mention more than one barrier.

Legend: NLU: non-Linux user, LU: Linux user.

fewer barriers. The output of the Fisher Exact Test for each barrier is shown in the final column in Table 2. As can be seen, none of these values are smaller than a critical level of $\alpha=.05$. Hence, we have to accept the null hypothesis that there is no significant difference between adopters and non-adopters with respect to any of the barriers.

We further investigated this relationship by considering whether organizations that mentioned a barrier exhibited a lower degree of OSSS assimilation than those organizations that did not report the barrier. To this end, a t-test for each barrier was conducted. None of these t-tests identified a significant difference at $\alpha=.05$.

\subsection{Relationship with Extent of Linux Adoption}

We also considered whether the extent to which the organization has adopted Linux had an influence on the barriers reported. The extent of Linux adoption was measured using a 7-point Likert scale, ranging from "no usage" to "to a very large extent". Based on this data, organizations were divided into two groups. The first group consisted of those organizations that did not make use of Linux, or only to a small extent (i.e., those who answered 1-3 on the 7-point Likert scale) and will be called "non-Linux users". The second group consisted of those organization who made use of Linux to at least a moderate extent (i.e., those who answered 4-7 on the 7-point Likert scale) and will be called "Linux users".

Table 3 shows a breakdown of the barriers reported by Linux and non-Linux users, as well as the frequency effect size for each barrier. Similar to before, the Fisher Exact Test was performed for each barrier to test whether the barriers reported differed based on the extent of Linux adoption. As can be seen in 
Table 3, none of the Fisher statistics are smaller than the critical value of $\alpha=.05$. Hence, we have found no significant difference between Linux and non-Linux users with respect to the barriers reported.

We subsequently performed a series of t-tests to investigate whether organizations that reported a barrier exhibited a lower extent of Linux adoption. Results showed only one significant difference at $\alpha=.05$. It was shown that organizations that reported that they could not identify any further barriers to the adoption of OSSS exhibited a higher extent of Linux adoption $(t=-2.028$, $d f=54, p=.048)$.

\section{Discussion and Conclusion}

Several of the barriers discussed above were also mentioned in previous research (see e.g., 10201112 25519]). However, an important result of this study is that the most important barrier to the further adoption of OSSS was insufficient knowledge on OSSS. Our data suggests that the lack of internal knowledge is more important than a lack of external knowledge. This can be explained by the fact that it can be rather difficult to reorient the knowledge base of the organization. Previous research has shown that the skills of employees are often brand-specific [29], or that the IT staff may resist a change towards a new platform if it goes against their "vested interests" 3033. The use of service providers is more flexible since they can be hired on an ad-hoc basis to support the organization in the adoption of OSSS.

Overall, our results indicate that there are important knowledge barriers involved in the adoption of OSSS. Knowledge barriers may occur during the adoption of knowledge-intensive technologies [1]. To overcome these barriers, organizations must engage in a process of organizational learning [17]. With respect to the adoption of OSSS, organizations may have to invest considerable learning effort if they have primarily experience with proprietary software. The assimilation of OSSS then becomes a process of organizational learning.

These results are very consistent with our findings from previous quantitative research that investigated which reasons influence the assimilation of OSSS [32]. Results showed that the assimilation of OSSS was primarily influenced by the knowledge available to organizations, which suggested the presence of knowledge barriers 32 . In addition, our previous research also showed that the availability of internal knowledge had a more important impact on the assimilation of OSSS than the availability of external knowledge [32. The most important factor influencing the assimilation of OSSS was the presence of boundary spanners in the organization 32 . Such boundary spanners are important in overcoming the knowledge barriers involved in the adoption of new technologies 4 . The findings of this study therefore provide further support for our previous conclusions. This is remarkable since the aim and research design of both studies was quite different.

We further used statistical techniques to analyze our qualitative data. Our results showed that organizations that have adopted Linux to a higher extent tend 
to report more often that they cannot identify any remaining barriers to the further adoption of OSSS. This suggests that barriers to adoption are only overcome in the final stages of the assimilation process. This illustrates the importance of also considering which barriers exist for organizations that have already adopted OSSS. In addition, we were not able to find any statistically significant indications that the barriers reported by organizations were related to their degree of OSSS assimilation or their extent of Linux adoption. This implies that these barriers remain an issue during the whole assimilation process. Hence, our data does not suggest that organizations are effectively able to overcome these barriers to adoption. This means, for example, that a lack of knowledge continues to be an important problem for organizations when increasing their assimilation of OSSS.

\subsection{Contributions}

The main contribution of this study is that it addresses a topic that has not been previously addressed in literature, namely the barries that exist to the further adoption of OSS. We provided an overview of which barriers exist to the adoption of OSSS by using a large-scale sample including both organizations that did not adopt OSSS, and organizations that have adopted OSSS to at least some extent. By investigating the relationship between the existence of these barriers and the degree to which the organization has adopted OSSS, it was shown that these barriers remain important in the whole assimilation process of OSSS. This highlights the importance of also considering the barriers that limit the further adoption of OSSS.

A second contribution is that we confirmed the results from our previous quantitative study. This is noteworthy since a very different approach was used in this paper. A first difference is that our previous study focused on determining the factors that influence the assimilation of OSSS. In our present study, we were concerned with which barriers inhibited the further use of OSSS. It has been noted in literature that adoption and non-adoption are two fundamentally different phenomena 219]. A second difference is that our previous study used quantitative techniques to analyze the data, while a combination of qualitative and quantitative techniques were used in this paper. The fact that the results from both studies using multiple methods are very consistent increases the (nomological) validity of our results.

An important practical implication of this study is that organizations should consider the adoption of OSSS to be a learning process. This learning process is required to overcome the knowledge barriers associated with the adoption of OSSS. This implies that organizations should invest sufficient internal resources to support this learning process, instead of relying exclusively on a service provider. By having sufficient internal knowledge, the assimilation of OSSS can be facilitated. Decision makers can also take initiatives to foster the acquisition and exploitation of internal knowledge. Since boundary spanners have found to be important in overcoming knowledge barriers, decision makers could try to stimulate the emergence of informal boundary spanners and seek their 
input during the adoption process. In addition, decision makers should be aware of the existence of these knowledge barriers when considering the adoption of OSSS. This means that they should not only consider the advantages that the adoption of OSSS could offer to the organization (e.g., lower cost, the availability of the source code, or the reduction of vendor lock-in), but should also take into account whether the organization has the ability to acquire the knowledge required to use OSSS.

\subsection{Limitations and Future Research}

This study has a few limitations that provide opportunities for future research. A first limitation is that we did not obtain the perception of each organization with respect to each of the barriers reported in Table 1. Instead, organizations were asked to report any perceived barriers in a free text field. This way, we only obtained those barriers that spontaneously came into the mind of the respondent, without further probing for their opinion on other barriers. This may have had an impact on our results. Future research may therefore take the list of barriers identified in this study as a starting point, and measure the perception of organizations towards each barrier. Finally, the external validity of our study is limited in the sense that the scope of our study was limited to Belgian organizations and OSSS. It would therefore be useful to replicate this study in other regions and by using a different set of OSS products (e.g., OSS desktop products such as OpenOffice.org) to see to which degree our results can be generalized.

\section{References}

1. Attewell, P.: Technology diffusion and organizational learning: The case of business computing. Organization Science 3(1), 1-19 (1992)

2. Auerbach, C.F., Silverstein, L.B.: Qualitative Data: An Introduction to Coding and Analysis. Qualitative Studies in Psychology. New York University Press, New York (2003)

3. Bazeley, P.: The contribution of computer software to integrating qualitative and quantitative data analyses. Research in the schools 13(1), 64-74 (2006)

4. Cohen, W.M., Levinthal, D.A.: Absorptive capacity: A new perspective on learning and innovation. Administrative Science Quarterly 35(1), 128-152 (1990)

5. Dedrick, J., West, J.: Why firms adopt open source platforms: A grounded theory of innovation and standards adoption. In: King, J.L., Lyytinen, K. (eds.) Proceedings of the Workshop on Standard Making: A Critical Research Frontier for Information Systems, Seattle, WA, December 12-14, pp. 236-257 (2003)

6. Fichman, R.G.: The diffusion and assimilation of information technology innovations. In: Zmud, R. (ed.) Framing the Domains of IT Management: Projecting the Future Through the Past, pp. 105-128. Pinnaflex Educational Resources, Cincinnati (2000)

7. Fichman, R.G., Kemerer, C.F.: The assimilation of software process innovations: An organizational learning perspective. Management Science 43(10), 1345-1363 (1997) 
8. Fitzgerald, B., Kenny, T.: Open source software in the trenches: Lessons from a large scale implementation. In: March, S.T., Massey, A., DeGross, J.I. (eds.) Proceedings of 24th International Conference on Information Systems (ICIS 2003), Seattle, WA, December 14-17, pp. 316-326. Association for Information Systems, Atlanta (2003)

9. Gatignon, H., Robertson, T.: Technology diffusion: An empirical test of competitive effects. Journal of Marketing 53(1), 35-49 (1989)

10. Goode, S.: Something for nothing: Management rejection of open source software in Australia's top firms. Information \& Management 42(5), 669-681 (2005)

11. Holck, J., Larsen, M.H., Pedersen, M.K.: Identifying business barriers and enablers for the adoption of open source software. In: Proceedings of the 13th International Conference on Information Systems Development, Vilnius, Lithuania, September 9-11 (2004)

12. Holck, J., Larsen, M.H., Pedersen, M.K.: Managerial and technical barriers to the adoption of open source software. In: Franch, X., Port, D. (eds.) ICCBSS 2005. LNCS, vol. 3412, pp. 289-300. Springer, Heidelberg (2005)

13. Huysmans, P., Ven, K., Verelst, J.: Reasons for the non-adoption of openoffice. org in a data-intensive public administration. First Monday 13(10) (2008)

14. Larsen, M.H., Holck, J., Pedersen, M.K.: The challenges of open source software in IT adoption: Enterprise architecture versus total cost of ownership. In: Proceedings of the 27th Information Systems Research Seminar in Scandinavia (IRIS27), Falkenberg, Sweden, August 14-17 (2004)

15. Li, Y., Tan, C.H., Teo, H.H., Siow, A.: A human capital perspective of organizational intention to adopt open source software. In: Avison, D., Galletta, D., DeGross, J.I. (eds.) Proceeding of the 26th Annual International Conference on Information Systems (ICIS 2005), Las Vegas, NV, December 11-14, pp. 137-149. Association for Information Systems, Atlanta (2005)

16. Lundell, B., Lings, B., Lindqvist, E.: Perceptions and uptake of open source in Swedish organisations. In: Damiani, E., Fitzgerald, B., Scacchi, W., Scotto, M., Succi, G. (eds.) Open Source Systems, IFIP Working Group 2.13 Foundation on Open Source Software, Como, Italy, June 8-10, 2006. IFIP International Federation for Information Processing, vol. 203, pp. 155-163. Springer, Boston (2006)

17. Miles, M.B., Huberman, A.M.: Qualitative Data Analysis: An Expanded Sourcebook, 2nd edn. Sage Publications, Thousand Oaks (1994)

18. Miralles, F., Sieber, S., Valor, J.: An exploratory framework for assessing open source software adoption. Systèmes d'Information et Management 11(1), 85-111 (2006)

19. Morgado, G., van Leeuwen, M., Özel, B., Erkan, K.: Current status of F/OSS (2007), http://www.tossad.org/content/download/1385/6894/file/ tOSSad_D18_V2.3.pdf

20. Morgan, L., Finnegan, P.: How perceptions of open source software influence adoption: An exploratory study. In: Österle, H., Schelp, J., Winter, R. (eds.) Proceedings of the 15th European Conference on Information Systems (ECIS 2007), St. Gallen, Switzerland, June 7-9, pp. 973-984. University of St. Gallen, St. Gallen, Switzerland (2007)

21. Nabih, M.I., Bloem, S.G., Poiesz, T.B.: Conceptual issues in the study of innovation adoption behavior. Advances in Consumer Research 24(1), 190-196 (1997)

22. Onwuegbuzie, A.J.: Effect sizes in qualitative research: A prolegomenon. Quality and Quantity 37(4), 393-409 (2003) 
23. Onwuegbuzie, A.J., Dickinson, W.B.: Mixed methods analysis and information visualization: Graphical display for effective communication of research results. The Qualitative Report 13(2), 204-225 (2008)

24. Onwuegbuzie, A.J., Teddlie, C.: A framework for analyzing data in mixed methods research. In: Tashakkori, A., Teddlie, C. (eds.) Handbook of Mixed Methods in Social and Behavioral Research, pp. 351-383. Sage Publications, Thousand Oaks (2003)

25. Paré, G., Wybo, M., Delannoy, C.: Barriers to open source software adoption in quebecs health care organizations. Journal of Medical Systems 33(1), 1-7 (2009)

26. Pett, M.A.: Nonparametric Statistics for Health Care Research: Statistics for Small Samples and Unusual Distributions. Sage Publications, Thousand Oaks (1997)

27. Saldaña, J.: The Coding Manual for Qualitative Researchers. Sage, Los Angeles (2009)

28. Sandelowski, M., Voils, C.I., Knafl, G.: On quantitizing. Journal of Mixed Methods Research 3(3), 208-222 (2009)

29. Shapiro, C., Varian, H.R.: Information Rules: A Strategic Guide to the Network Economy. Harvard Business School Press, Boston (1999)

30. Swanson, B.E.: Information systems innovation among organizations. Management Science 40(9), 1069-1092 (1994)

31. Ven, K., Verelst, J.: The organizational adoption of open source server software by Belgian organizations. In: Damiani, E., Fitzgerald, B., Scacchi, W., Scotto, M., Succi, G. (eds.) Open Source Systems, IFIP Working Group 2.13 Foundation on Open Source Software. IFIP International Federation for Information Processing, vol. 203, pp. 111-122. Springer, Boston (2006)

32. Ven, K., Verelst, J.: The organizational adoption of open source server software: A quantitative study. In: Golden, W., Acton, T., Conboy, K., van der Heijden, H., Tuunainen, V. (eds.) Proceedings of the 16th European Conference on Information Systems (ECIS 2008), Galway, Ireland, June 9-11, pp. 1430-1441 (2008)

33. Zmud, R.W.: Diffusion of modern software practices: Influence of centralization and formalization. Management Science 28(12), 1421-1431 (1982) 